\title{
Musik zwischen Nation Building und Internationalität. Italien um 1900
}

\author{
MARKUS ENGELHARDT, ROM
}

Das moderne italienische Königreich, das Regno d'Italia zwischen 1861 und 19461, umfasst nicht nur die Zeit des Faschismus, sondern auch zwei Weltkriege. Was seine Musik und deren gesellschaftliche und politische Kontextualisierung anbelangt, ist mithin die Expertise musikgeschichtlicher Konfliktforschung besonders gefragt, der jüngst auch eine GfM-Jahrestagung ${ }^{2}$ gewidmet war. Nicht minder bedeutungsvoll ist jedoch seine erste Phase, die der staatlichen Einigung und des Nation Building, der Formierung des Landes zur Nation mit Stimme im Konzert der Nationen Europas.

Die Konkurrenz um politischen und kulturellen Prestigegewinn und die Konflikte unter den sich im 19. und 20. Jahrhundert profilierenden Nationalstaaten hängen eng miteinander zusammen. Italien erlangte 1861 staatliche Souveränität, die es in den kommenden Jahrzehnten zu rechtfertigen galt, und zwar zu allererst durch die Realisierung gesellschaftlicher und kultureller Einheit. Die Einheit wurde als Garant vor allem auch für das Bestehen der Nation auf internationaler Ebene propagiert.

Eine Nation außerhalb des internationalen Wettbewerbs ist schwer vorstellbar, Selbstbehauptung kultureller Identität im internationalen Kontext gehört zu den Bestimmungen und Wesensmerkmalen einer Nation. Allerdings wird internationale Konkurrenz, das gilt für politische, wirtschaftliche, militärische Aspekte ebenso wie für kulturelle, mit ungleichen Waffen ausgefochten und auch was die Bewertung kultureller Leistungen anbelangt, gibt es Hierarchien sowie Nationen, die kulturellen Primat beanspruchen, und andere, die diesem Primat unterworfen werden.

Was deutsche Musikdiskurse im Vorfeld des weltweiten politischen Konflikts des Ersten Weltkriegs anbelangt, so spricht aus ihnen eine gewisse Sorge, Italien - Land des Belcanto, im Fach anspruchsvoller Instrumental- und Kirchenmusik notorischer Nachzügler - könnte sich nicht nur zu einer politischen, sondern erneut auch zu einer musikalischen Großmacht entwickeln. So fragte um die Jahrhundertwende in den Sammelbänden der Internationalen

1 Die vorliegenden Ausführungen eröffneten auf der Jahrestagung der Gesellschaft für Musikforschung in Paderborn/Detmold (23.-26. September 2019) das Round Table "National - international: Musikverständnis und Musikpraxis im Umfeld politischer Konflikte". Für ihre Statements sei an dieser Stelle den weiteren Gesprächsteilnehmern gedankt: Boris Belge („Das ,deutsche Erbe' im sowjetischen Musikschaffen“), Dietrich Helms ("HulaMädchen und Capri-Fischer. Internationaler Sehnsuchtsort einer nationalen Schlagerproduktion in den Jahren um den Zweiten Weltkrieg") und Stefan Hanheide ( in Zeiten des Chauvinismus").

2 Jahrestagung der Gesellschaft für Musikforschung 2018 (25.-28. September) in Osnabrück mit dem Themenschwerpunkt 1 "Der Erste Weltkrieg in der musikwissenschaftlichen Forschung". 
Musikgesellschaft - sie liegen dieser Bestandsaufnahme zugrunde - der aus New Jersey stammende, aber in Deutschland aufgewachsene Musikwissenschaftsstudent und spätere Leiter der Musikabteilung der Library of Congress Oskar George Sonneck: „Wer weiß, ob nicht Italien doch noch einmal, auch in der Musik eine Großmacht würde!"3

Begonnen hatten Sonnecks Ausführungen mit der Feststellung, "Italien steht unter den Musikländern nicht mehr an erster Stelle, sondern reiht sich erst hinter Deutschland, Österreich-Ungarn, Belgien, Frankreich, England, Nordamerika, Norwegen, Rußland und anderen Kulturstaaten ein"4, womit dieser Musikdiskurs Denkmustern der Zeit folgend Hierarchien voraussetzt, wie sie für nationalstaatliche Konkurrenz generell bestimmend sind. Welches sind die Maßstäbe und Kriterien derartiger Hierarchisierungen?

In dem Panorama, das Sonneck um 1900 zur Musiknation Italien entfaltet, sind mehrere wiederkehrende Motive zu erkennen: (1) die Nähe und Ferne des italienischen zum deutschen Musikstandard. So werden deutsche Einflüsse auf Komponisten wie Rossini, Spontini, Cherubini und selbst Pacini hervorgehoben. (2) Deutsch-italienische Lehrer-Schüler-Verhältnisse sind von großer Bedeutung: der Bülow/Rheinberger-Schüler Giuseppe Buonamici, der RheinbergerSchüler Wolf-Ferrari, der Haberl-Schüler Lorenzo Perosi, der Reinecke-Schüler Luigi Torchi (mit seiner verblüffenden Kenntnis deutscher Romantiker), der Joachim-Schüler Ettore Pinelli usw. (3) Ausbildungswege, die Künstler wie Antonio Bazzini über Deutschland führten oder auch das Engagement deutscher Komponisten, Dirigenten, Pianisten etc. für das Musikleben in Italien: Hans von Bülow in Florenz, Martin Röder in Mailand. (4) Ein Hauptindikator für das Niveau des ausländischen Musiklebens ist der Anteil ,deutscher' Komponisten in Konzertprogrammen (Joseph Haydn, Ludwig van Beethoven, Carl Maria von Weber, Richard Wagner) und (5) eine ähnliche Aufwertung ist mit der Tatsache assoziiert, dass sich italienische Komponisten mit den Werken deutscher Komponisten befassen bzw. deren Stilhöhe und persönliche Größe zu erlangen suchen. Hierzu werden Verdis Studien von Bachs Wohltemperiertem Klavier und Wagners Lohengrin bemüht. Derselben Tendenz folgen Feststellungen zur Repertoirebildung, wie sie Sonneck zum dem Pianisten „G. Andreoli", i.e. Guglielmo Andreoli junior, trifft, der sein Publikum „bis Brahms zu führen" ${ }^{5}$ suche, oder in einem zeitlich benachbarten Beitrag die Bemerkungen Friedrich Spiros zu Alessandro Vessella, der an der Spitze der Banda Municipale Roms „seinen Quiriten [...] deutsche, aber auch neufranzösische und altitalienische Musik" beibringt. $^{6}$ Spiros bissige Insider-Reportagen zum römischen Musikleben dieser Jahre bedienen virtuos die gesamte Klaviatur anti-italienischer Ressentiments. Zugleich verdichtet sich hier

3 O. G. Sonneck, „Zum Wiederaufschwung des italienischen Musiklebens”, in: SIMG 1 (1899-1900), S. 630-670, hier S. 670.

4 Ebd., S. 630.

5 Ebd., S. 663.

6 Friedrich Spiro, „Musik in Rom”, in: ZIMG 1 (1899-1900), S. 189-201, hier S. 201. 
ähnlich wie in unserem Ausgangstext von George Sonneck das Spannungsverhältnis zwischen nationaler Identität und internationaler Konkurrenz. ${ }^{7}$

Aus der kulturellen Hierarchie, das ist ein weiterer Leitgedanke der von uns herangezogenen Zeitschriftenbeiträge, ergibt sich wie zwangsläufig eine Dynamik, mit der die überlegene nördliche Musikkultur die ihr unterlegene verdrängt. Italien wird im Grunde nur dort Bedeutung zuerkannt, wo es mit seiner Musik - wie im Falle von Weckmanns Collegium Musicum in Hamburg ${ }^{8}$ - dem deutschen Genie (Händel) Gelegenheit zu Anschluss und Vollendung bietet. Die Bedeutung der italienischen Oper des 17. und 18. Jahrhunderts scheint aus deutscher Sicht überhaupt nur darin zu liegen, dass diese durch Christoph Willibald Gluck, Wolfgang Amadeus Mozart und Richard Wagner überwunden wurde. Selbst der enorm kenntnisreiche

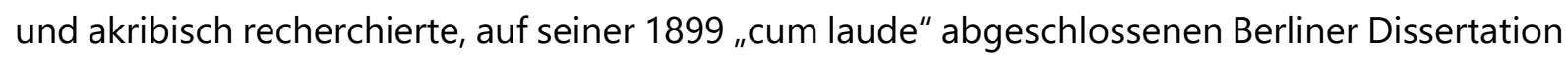
beruhende Beitrag von Albert Mayer-Reinach, "Carl Heinrich Graun als Opernkomponist", kommt nicht ohne dieses Überwindungsmotiv aus. Im letzten Moment und reichlich unvermittelt stellt er fest, dass das - überwiegend italienische bzw. aus Adaptionen italienischer und französischer Vorlagen bestehende - musiktheatralische Schaffen des preußischen Hofkapellmeisters der Vergessenheit anheimgefallen sei, bedingt durch den sich in den Neuerungen von Glucks Orfeo anbahnenden Sturz der italienischen Oper. ${ }^{9}$ Für Hugo Goldschmidt bereitete die italienische Oper immerhin den „Boden“, auf dem Händels Orchester Gestalt gewinnen konnte $^{10}$ während Hermann Kretzschmar ihren Erfolg mit der Unterhaltungssucht verkommener deutscher Fürstenhöfe erklärt und als eine auf niederes Kulturgut verlorene Verschwendung brandmarkt, welche "Volksfreunde und Patrioten [...] immer wieder beklagt" haben. ${ }^{11}$ Immerhin spielte für Kretzschmar die Unterhaltungskunst italienische Oper die positive Rolle einer Geburtshelferin der großen ,deutschen' Instrumentaltradition - (, unsere Wiener Klassik verdanken wir der Opernliebe der österreichischen Kaiser"12 ), womit auch hier wieder die glückliche Wendung der Ablösung eines südlichen Gattungstiefs durch eine nördliche Hochphase konstatiert wird.

Nationale Musikdiskurse um 1900 rekurrieren auf Gattungen als Stigmata (ausländische) bzw. als Gütesiegel (eigene Musikkultur) und hierbei vor allem auf die Polarität italienische Oper versus deutsche Kirchenmusik. Der deutschen Hofmusiker Johann Christian Bach, der nach

7 Aus Spiros Sicht vereinen sich in Rom piemontesische Bürokraten mit dem „Pöbel“ im Genuss niedriger Kultur (Koloraturgesang, Ballett usw.), „eine Bourgeoisie im germanischen, französischen oder russischen Sinne existiert nicht" (ebd., S. 191). Bologna allein habe engere Fühlung mit ausländischer Bildung (die sich z. B in Martuccis Chor- und Orchesterkonzerten niederschlage). In der Hauptstadt hingegen werde Musik deutscher Provenienz Opfer ,chauvinistisch' ausgeblendet.

8 Max Seiffert, "Matthias Weckmann und das Collegium Musicum in Hamburg. Ein Beitrag zur deutschen Musikgeschichte des 17. Jahrhunderts", in: SIMG 2 (1901-1902), S. 76-132.

9 Albert Mayer-Reinach, "Carl Heinrich Graun als Opernkomponist”, in: SIMG 1 (1899-1900), S. 446-529.

10 Hugo Goldschmidt, "Das Orchester der italienischen Oper im 17. Jahrhundert”, in: SIMG 2 (1900-1901), S. 16-75.

11 Hermann Kretzschmar, „Das erste Jahrhundert der deutschen Oper”, in: SIMG 3 (1901-1902), S. $270-293$.

12 Ebd., S. 293. 
Mailand geht, dort zum katholischen Glauben übertritt und Domkapellmeister wird, wird Max Schwarz zufolge durch die italienische Oper jeglicher Voraussetzung beraubt, anständige Kirchenmusik zu komponieren. Heinses Roman Hildegard von Hohenthal in den Zeugenstand rufend stellt Schwarz in seinem Beitrag "Johann Christian Bach (1735-1782)“ fest: „Bach komponierte ohne einen Funken Glauben und zu prunkvoll. Seine Kompositionen dieser Gattung sind nicht Herzenssache, und er kann den Opern-Komponisten nicht verleugnen." ${ }^{13}$ Er "hatte nicht die Kraft in sich, mit den alten Traditionen der Oper zu brechen, und vielleicht darf ich sagen, nicht die Gelegenheit. Sein Leben brachte er fern von deutscher Gesinnung und Empfindung zu. ${ }^{14}$ Als Gegenbeispiel lernen wir aus einem anderen Beitrag, dass auf die Kirchenstücke eines Johann Kuhnau (hat Italien „musikalisch durchaus nicht geschätzt”) von dessen italienischem Lehrer Vincenzo Albrici „weder die lebhafte Dramatik noch die echt italienische Melodik [...] übergegangen" sei. ${ }^{15}$

Nicht zu überhören sind in den Musikdiskursen, wie sie die Periodika der Internationalen Musikgesellschaft um 1900 festhalten, auch ,deutsche' Stimmen, die der Musiknation Italien große Bewunderung zollen, und dies sowohl aus aktueller wie historischer Perspektive. Für letztere mag Friedrich Ludwig stehen, der für das Trecento und noch mehr für das 15. Jahrhundert die Bedeutung Italiens als Sammelbecken der unterschiedlichsten nationalen Musikstile hervorhebt. Ludwig attestiert dem "für die Musik auch des Auslands so dankbaren Italien"16 mithin eine geschichtlich begründete Internationalität.

Das aktuelle Beispiel ist Verdi. In dem bereits zitierten Nachruf spricht Hermann Abert von der "Nationaltrauer" der "Stammesgenossen"17 Verdis, von der Lücke, die der "allseits anerkannte Führer" hinterlassen habe und die keiner der zurückbleibenden "kleineren Diadochen"18 werde füllen können. Verdi ist aus deutscher Sicht, und da macht auch der sonst so polemische Rom-Beobachter Friedrich Spiro keine Ausnahme, die vollkommene Verschmelzung von Musik und Nation; „in der That", so Spiro, sei "diese Liebe zu Verdi, welche jeden zurechnungsfähigen Italiener vom Carabiniere herab bis zum Minister beseelt, einer der höchsten Ehrentitel der Nation; in diesem Punkte können alle anderen von ihr lernen" ${ }^{19}$ Nationalkomponisten wie Verdi genießen ungeteilte Wertschätzung über nationale Abgrenzungen und Konkurrenzen des internationalen Prestigestrebens hinweg - oder anders formuliert: Ihre nationale Symbolkraft ist internationaler Konsens. Und so bestätigt auch der nationale Musikdiskurs um 1900

13 Max Schwarz, „Johann Christian Bach (1735-1782)”, in: SIMG 2 (1900-1901), S. 401-454, hier S. 416.

14 Ebd., S. 417.

15 Richard Münnich, „Kuhnau's Leben“, in: SIMG 3 (1901-1902), S. 473-527, hier S. 490.

16 Friedrich Ludwig, "Die mehrstimmige Musik des 14. Jahrhunderts”, in: SIMG 4 (1902-1903), S. 16-69, hier S. 68.

17 Hermann Abert, "Giuseppe Verdi", in: ZIMG 2 (1900-1901), S. 204-207, hier S. 204.

18 Ebd., S. 207.

19 Friedrich Spiro, „Römisches Musikleben im Jahre 1901”, in: ZIMG 3 (1901-1902), S. 14-19, hier S. 16. 
manche grundsätzliche Erkenntnis der aktuellen musikwissenschaftlichen Friedensforschung: Musik ist wie jede Kunst eine für ideologische Funktionalisierungen anfällige Kunst, aber sie ist und bleibt in Zeiten nationalen Eiferns und selbst politischer Konflikte und Kriege immer auch die Kunst der Versöhnung und Konsens stiftender Potentiale.

Zitation: Markus Engelhardt, "Musik zwischen Nation Building und Internationalität. Italien um 1900", in: Freie Beiträge zur Jahrestagung der Gesellschaft für Musikforschung 2019, hrsg. von Nina Jaeschke und Rebecca Grotjahn (= Musikwissenschaft: Aktuelle Perspektiven. Bericht über die Jahrestagung der Gesellschaft für Musikforschung 2019 in Paderborn und Detmold, Bd. 1), Detmold 2020, S. 109-114, DOI: 10.25366/2020.54. 


\section{Abstract}

In this article German contributions to periodicals of the International Musicological Society focussing on Italian musical life in Italy around 1900 are analyzed as testimonies of Italy's new importance as a music nation at that time. The German perspective on musical culture in the Kingdom of Italy follows hierarchies that are closely linked to political and economic rivalry between the two nations. At different levels (music education, formation of composers and musicians, local repertories, musical genres) well-known concepts of German supremacy can be recognized. Nevertheless, the national music debates include also phenomena which strongly confirm music as art of great potential for international consensus.

\section{Kurzvita}

Markus Engelhardt war wissenschaftlicher Mitarbeiter an der Hochschule für Musik in Frankfurt am Main und an der Universität Bayreuth (Forschungsinstitut für Musiktheater). AnschlieBend hatte er die Leitung des Deutschen Studienzentrums in Venedig inne. Seit 1997 ist er Leiter der Musikgeschichtlichen Abteilung des Deutschen Historischen Instituts in Rom. Sein Forschungsschwerpunkt ist die Italienische Musik des 19. und 20. Jahrhunderts. 


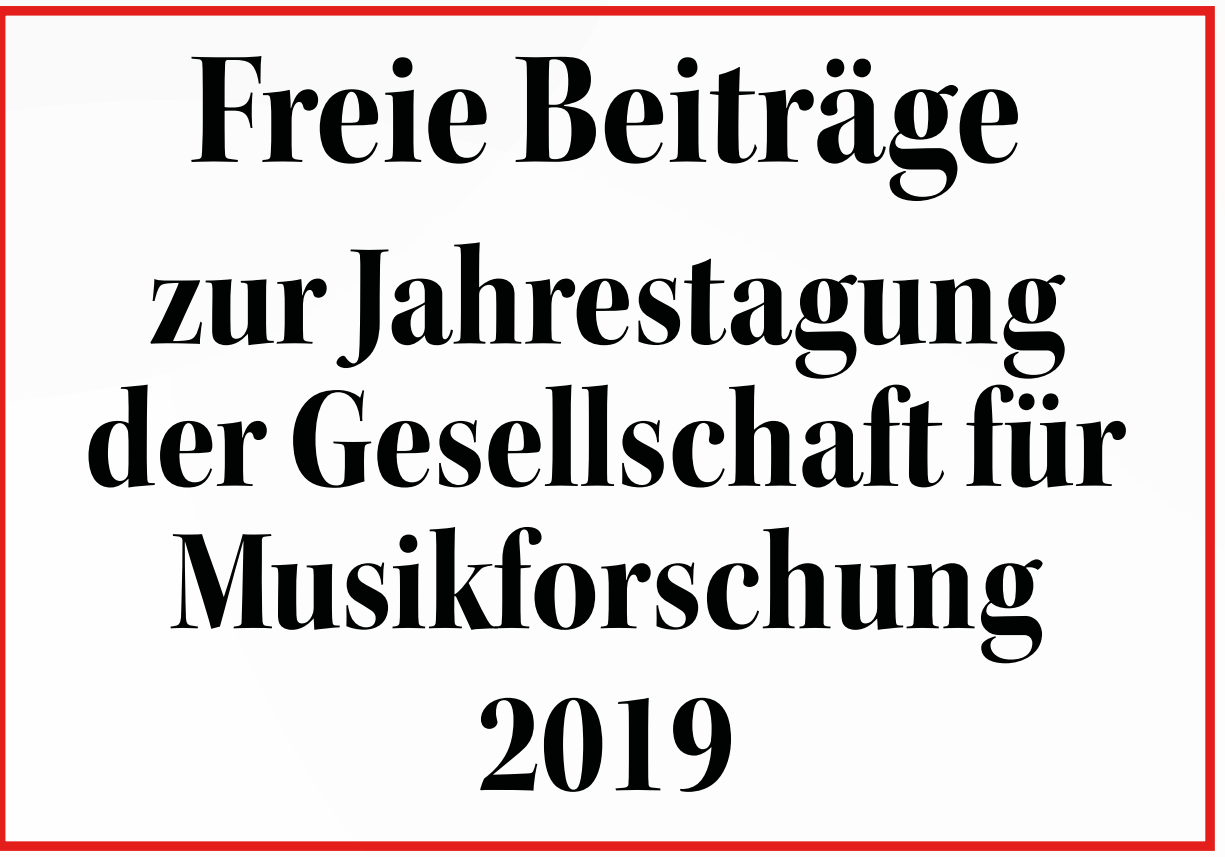

Herausgegeben von Nina Jaeschke und Rebecca Grotjahn

Musikwissenschaft: Aktuelle Perspektiven 1 
Freie Beiträge 


\section{Musikwissenschaft: Aktuelle Perspektiven}

Bericht über die Jahrestagung der Gesellschaft für Musikforschung 2019 in Paderborn und Detmold

Herausgegeben von Rebecca Grotjahn und Nina Jaeschke

Band 1 


\section{Freie Beiträge}

\section{zur Jahrestagung der Gesellschaft für Musikforschung 2019}

Herausgegeben von Nina Jaeschke und Rebecca Grotjahn

Detmold: Musikwissenschaftliches Seminar der Universität Paderborn und der Hochschule für Musik Detmold 2020 
DOI: $10.25366 / 2020.42$

Online-Version verfügbar unter der Lizenz: Urheberrecht 1.0, $<$ https://rightsstatements.org/page/InC/1.0/?language=de>

Bibliografische Information der Deutschen Nationalbibliothek

Die Deutsche Nationalbibliothek verzeichnet diese Publikation in der Deutschen Nationalbibliografie; detaillierte bibliografische Daten sind im Internet über http://dnb.d-nb.de abrufbar.

\section{Impressum}

Redaktion: Nina Jaeschke, Rebecca Grotjahn und Jonas Spieker Satz: Nina Jaeschke

(C) Musikwissenschaftliches Seminar der Universität Paderborn und der Hochschule für Musik Detmold 2020 


\section{INHALT}

Vorwort $\quad$ IX

Komponieren für das Radio: Akteure, Diskurse, Praktiken $\quad 1$

Musikwissenschaft - Feminismus - Kritik: Ein Generationenaustausch 6

\section{Stefan Alschner}

Der Wagner-Sänger Joseph Aloys Tichatschek - Vom Nachlass zum Netzwerk

\section{Alenka Barber-Kersovan}

Songs for the Goddess. Das popmusikalische Neo-Matriarchat zwischen Ethno-Beat,

erfundenen Traditionen und kommerzieller Vermarktung

Elias Berner, Julia Jaklin, Peter Provaznik, Matej Santi, Cornelia Szabó-Knotik

Musikgeschichte anders erzählen? Das Beispiel der 1970er in Österreich.

Musikhistoriographie in der Zeit der Digitalisierung

\section{Mauro Fosco Bertola}

„Ein Laut so klagevoll”. Lohengrin zwischen Richard Wagner und Salvatore Sciarrino

\section{Matthieu Cailliez}

Europäische Rezeption der Berliner Hofoper und Hofkapelle von 1842 bis 1849

\section{lacopo Cividini}

Zwischen klassischer Musikphilologie und angewandter Informatik:

Die Digitale Mozart-Edition (DME) der Stiftung Mozarteum Salzburg

\section{Marko Deisinger}

Fortschrittliche Technologie im Dienste eines Antimodernisten.

Heinrich Schenker und der österreichische Rundfunk

\section{Norbert Dubowy}

Vom Kritischen Bericht zur Kritischen Dokumentation am Beispiel der Digital-interaktiven Mozart-Edition

\section{Markus Engelhardt}

Musik zwischen Nation Building und Internationalität. Italien um 1900

\section{Maryam Haiawi}

Das Oratorium im Spannungsfeld der Konfessionen: 


\section{Judith I. Haug}

"Manch eine*r liegt, morgens noch trunken, im Rosengarten" - Rekonstruktionen

osmanischer Musikgeschichte in Gesangstextsammlungen

\section{Renate Koch}

Marcel Prawy und das erste Broadway-Musical im Österreich der Nachkriegszeit

Susanne Kogler, Julia Mair, Juliane Oberegger, Johanna Trummer

Erich Marckhl - Musikausbildung in der Steiermark nach 1945.

Brüche und Kontinuitäten

\section{Marie-Anne Kohl}

Die weinende Jury. "Geschlechtslose" Tränen bei globalen Musik-Castingshows?

\section{Fabian Kolb}

Tanztheater und filmische Ästhetik. Cineastische Einflüsse und Gestaltungsweisen in den Kompositionen für die Ballets Suédois 1920-1925

\section{Christian Lehmann}

Tempobezeichnungen von Julius Stockhausen für Die schöne Müllerin:

Ein Quellenfund

\section{Martin Link}

Signum et gens - Zur Gendersemiotik in Clara und Robert Schumanns Liederzyklus Liebesfrühling

\section{Livio Marcaletti}

„Strafspiel" und satirische Stilmittel in musikdramatischen Gattungen des frühen 18. Jahrhunderts

\section{Tobias Marx, Martin Lissner}

Thüringer Musikszene - Jugendmusikredaktionen als außerschulische musikbezogene Bildungskontexte

\section{Maho Naito}

Die Parallelität der Entstehungsprozesse der ersten beiden Symphonien Gustav

Mahlers: Instrumentation, Revision und Dirigierpraxis

\section{Elisa Novara}

Eine Schumann-Werkstatt? Zur Übertragbarkeit der Methoden vom Projekt 
Theodora Oancea, Joachim Pollmann, Jonas Spieker

Kollaborateure - Involvierte - Profiteure. Erarbeitung eines Online-Lexikons zur

Musik in der NS-Zeit

\section{Kiron Patka}

„Ich wollte eigentlich Sängerin werden." Berufsselbstbilder von Tontechniker*innen im Radio

\section{Siegwart Reichwald}

Die Leiden der jungen Clara: Das Klaviertrio Opus 17 als Ausdruck einer Neu-

Romantikerin

\section{Elisa Ringendahl}

Lied versus Oper - Pole musikalischer Gattungen bei Oscar Bie

\section{Benedikt Schubert}

Struktur und Exegese. Über Eigentümlichkeiten in der Arie "Des Vaters Stimme ließ sich hören" (BWV 7/4)

Uwe Seifert, Sebastian Klaßmann, Timo Varelmann, Nils Dahmen

Computational Thinking in der Musikwissenschaft: Jupyter Notebook als Umgebung

für Lehre und Forschung

\section{Yusuke Takamatsu}

Synthese als Modus der Prozessualität bei Schubert:

Sein spezifisches Wiederholungsprinzip im langsamen Satz

\section{Daniel Tiemeyer}

Johann Nepomuk Hummels Sonate in fis-Moll Op. 81 - Studien zu Entstehungs-

hintergrund, Rezeption und formalerStruktur

\section{Andrea van der Smissen}

Musikalische Innovation im Umfeld der Moderne und historischen Avantgarde in Ungarn

\section{Tim Ziemer, Holger Schultheis}

Psychoakustische Sonifikation zur Navigation in bildgeführter Chirurgie

\section{Magdalena Zorn}

Musik mit dem Radio hören: Über den Begriff der musikalischen Aufführung 
Gabriele Buschmeier in memoriam 


\section{Vorwort}

Die vorliegenden Bände dokumentieren die Jahrestagung der Gesellschaft für Musikforschung 2019. In den dreieinhalb Tagen vom 23. bis zum 26. September 2019 wurden in Paderborn und Detmold nicht weniger als 185 Beiträge präsentiert, verteilt auf diverse Symposien, Round tables, Freie Sektionen und Postersessions. Sie alle auf einen Nenner bringen zu wollen, ist ein Ding der Unmöglichkeit - und das ist gut so, ist es doch Ziel der Jahrestagungen, die große Vielfalt der Themen und Methoden des Faches Musikwissenschaft abzubilden. Um die thematische Vielfalt der freien Referate angemessen abbilden zu können und gleichzeitig den inhaltlichen Schwerpunkten der beiden hier publizierten Hauptsymposien ausreichend Raum bieten zu können, erscheinen diese in drei Bänden.

„Musikwissenschaft: Aktuelle Perspektiven": Der Titel der kleinen Reihe ist keine Verlegenheitslösung. Musikwissenschaft im Kontext der Digital Humanities; Musikwissenschaft und Feminismus; Musik und Medien; Musikalische Interpretation - schon die Felder, die von den vier Hauptsymposien bespielt wurden, wären noch vor wenigen Jahrzehnten allenfalls an der Peripherie das Faches zu finden gewesen. Sie entsprechen Arbeitsschwerpunkten der Lehrenden am Musikwissenschaftlichen Seminar der Universität Paderborn und der Hochschule für Musik Detmold, das die Tagung ausrichtete. Zugleich nehmen sie Bezug auf aktuelle Ereignisse und Entwicklungen. So erwuchs das von Andreas Münzmay und Joachim Veit organisierte Symposium „Brückenschläge - Informatik und Musikwissenschaft im Dialog" unmittelbar aus den Erfahrungen im Virtuellen Forschungsverbund Edirom (ViFE) und im fakultäten- und hochschulübergreifenden Zentrum Musik-Edition-Medien (ZenMEM). Der 200. Geburtstag von Clara Wieck/Schumann war der Anlass für das von Rebecca Grotjahn geleitete Symposium „Die Begleiterin - Clara Schumann, Lied und Liedinterpretation", das in enger Kooperation mit der Hochschule für Musik Detmold durchgeführt wurde. Das Hauptsymposium „Brückenschläge" wird in einem separaten Band publiziert (Bd. 3 der vorliegenden Reihe). Im Rahmen dieses Symposiums führte die von Stefanie Acquavella-Rauch geleitete Fachgruppe Digitale Musikwissenschaft eine Posterpräsentation durch, die von den Beiträger*innen erfreulicherweise zu kürzeren Texten umgearbeitet wurden, sodass sie hier ebenfalls, zusammen mit den Postern,

publiziert werden können. Hinzu kommen einige Beiträge, die bereits bei der Jahrestagung 2018 in Osnabrück präsentiert wurden. Auch das Hauptsymposium "Die Begleiterin" wird in einem eigenen Band (Bd. 2) publiziert. Die Beiträge zu den beiden anderen Hauptsymposien hingegen werden an anderen Orten veröffentlicht; in Band 1 („Freie Beiträge zur Jahrestagung der Gesellschaft für Musikforschung 2019") der vorliegenden Publikation finden sich jedoch Einführungen und Abstracts. Das Symposium „Komponieren für das Radio" unter Leitung von Antje Tumat und Camilla Bork (Katholieke Universiteit Leuven) behandelte Einflüsse des Mediums auf Kompositionsprozesse sowie durch radiophone Kompositionen bzw. radiophonen Klang ausgelöste Diskurse. Sarah Schauberger und Cornelia Bartsch (Universität Oldenburg) nahmen das 25-jährige Jubiläum der Fachgruppe Frauen- und Genderstudien zum Anlass für einen Generationenaustausch zum Thema "Musikwissenschaft - Feminismus - Kritik": Was wa- 
ren vor einem Vierteljahrhundert Inhalte und Aufgaben einer feministischen Musikwissenschaft und wie kann sich diese heute positionieren?

Bewusst haben wir im Tagungsbericht auf inhaltliche Eingriffe in die Beiträge verzichtet. ${ }^{1}$ Das gilt besonders für die Freien Referate: Es galt, den Charakter der Jahrestagung als Forum für ,freie', d. h. innovative und auch experimentelle Gedanken zu wahren. Einige Kolleg*innen, die die Tagung mit Vorträgen und Posterpräsentationen bereichert hatten, haben sich gegen eine Publikation im vorliegenden Band entschieden - sei es, weil sie eine Möglichkeit fanden, ihre Beiträge in einem inhaltlich passenderen Rahmen zu veröffentlichen, sei es, weil ihre Überlegungen in ihre entstehenden Qualifikationsschriften fließen sollen, oder sei es, weil sie von den Autor*innen in der vorgetragenen Form zunächst verworfen wurden. Auch damit erfüllt eine Freie-Referate-Sektion ihren Zweck: Die Diskussionen mit der versammelten Fach-Öffentlichkeit sollen dabei helfen, Gedanken weiterzuentwickeln und zu verändern. In diesem Sinne sei allen Beteiligten - den Autor*innen, den nichtpublizierenden Referent*innen und den Mit-Diskutant*innen - ganz herzlich gedankt für ihr Mitwirken bei der Tagung.

Unser herzlicher Dank gilt einer Reihe weiterer Personen, die zum Gelingen dieser drei Bände beigetragen haben. Hier ist besonders Jonas Spieker zu nennen, der uns tatkräftig bei der Redaktion geholfen hat. Andrea Hammes (SLUB Dresden) sei herzlich für die Aufnahme unseres Bandes auf musiconn.publish gedankt - wir freuen uns, damit unsererseits zur Etablierung dieser innovativen Publikationsplattform beizutragen.

Erneut möchten wir an dieser Stelle allen Menschen danken, die uns bei der Organisation, Ausrichtung und Finanzierung der Tagung selbst unterstützt haben: der Präsidentin der Universität Paderborn, Prof. Dr. Birgitt Riegraf, dem Rektor der Hochschule für Musik Detmold, Prof. Dr. Thomas Grosse, den Kolleginnen und Kollegen der beiden beteiligten Hochschulen, dem Vorstand der Gesellschaft für Musikforschung, der Universitätsgesellschaft Paderborn und allen Sponsoren. Besonders dankbar sind wir den Mitarbeiter*innen und den studentischen bzw. wissenschaftlichen Hilfskräften des Musikwissenschaftlichen Seminars, die bei der Vorbereitung und Ausrichtung der Tagung immensen Einsatz zeigten - stellvertretend sei an dieser Stelle Johanna Imm erwähnt, die zusammen mit Nina Jaeschke das Herz des Organisationsteams bildete.

Wir widmen diese Reihe Dr. Gabriele Buschmeier, dem langjährigen Vorstandsmitglied der Gesellschaft für Musikforschung, die kurz vor der Publikation dieses Bandes unerwartet verstarb.

Detmold, im September 2020

Rebecca Grotjahn und Nina Jaeschke

Zitation: Rebecca Grotjahn und Nina Jaeschke, „Vorwort”, in: Freie Beiträge zur Jahrestagung der Gesellschaft für Musikforschung 2019, hrsg. von Nina Jaeschke und Rebecca Grotjahn (= Musikwissenschaft: Aktuelle Perspektiven. Bericht über die Jahrestagung der Gesellschaft für Musikforschung 2019 in Paderborn und Detmold, Bd. 1), Detmold 2020, S. IX-X, DOI: 10.25366/2020.43.

1 Freigestellt war den Autor*innen auch, ob sie sich für eine gendersensible Sprache entscheiden bzw. welche Form des Genderns sie bevorzugen. 